\title{
Higher Cell Viability and Enhanced Sample Quality Following Laser-Assisted Liposuction versus Mechanical Liposuction
}

\author{
Alexander Levenberg ${ }^{1 *}$, Mickey Scheinowitz ${ }^{2}$, Orna Sharabani-Yosef ${ }^{2}$ \\ ${ }^{1}$ Department of Plastic Surgery, Tel Aviv Sourasky Medical Center, Tel Aviv, Israel \\ ${ }^{2}$ Department of Biomedical Engineering, Faculty of Engineering, Tel Aviv University, Tel Aviv, Israel \\ Email: ${ }^{*}$ leveplas@netvision.net.il
}

Received 3 August 2015; accepted 11 September 2015; published 14 September 2015

Copyright (C) 2015 by authors and Scientific Research Publishing Inc.

This work is licensed under the Creative Commons Attribution International License (CC BY). http://creativecommons.org/licenses/by/4.0/

(c) (†) Open Access

\begin{abstract}
Background: Despite the popularity of autologous fat transfer applications, high resorption rates, and consequential volume loss, have been reported. Viable adipocyte content has been defined as a key determinant of fat transfer longevity. Moreover, traces of blood, free oil fat and fibrotic tissue accelerate adipocyte degradation. Objective: To compare the effectiveness of a $1470 \mathrm{~nm}$, radial emitting laser-assisted liposection device to a mechanical liposection device in maintaining adipocyte viability in fat tissue harvests. Methods: Bilateral subcutaneous adipose tissue samples were harvested from ten female patients. Fat was harvested from one side using the LipoLife laser-assisted liposuction device and from the other side with a Byron mechanical aspirator. Samples were visually analyzed and blood:fat ratios and cell viability were determined. Results: Laser-harvested samples separated into two distinct phases, with a negligible blood phase at the bottom (1.1\%) and a significant adipose phase at the top (98.9\%), containing small, uniform-sized cells, of which $95.7 \% \pm 2.7 \%$ proved viable. Mechanically harvested samples separated into blood $(18 \%)$, adipose $(60 \%)$ and lipid $(22 \%)$ phases. The adipose phase contained significant amounts of connective tissue, large adipose tissue fragments, large oil droplets and a mean $79.7 \% \pm 18.3 \%$ viable adipocytes. Conclusions: Laser liposuctioning was superior to mechanical liposuctioning, providing both higher cell viability and enhanced sample quality. The $1470 \mathrm{~nm}$ diode laser bears the potential of improving long-term clinical outcomes of fat transfer procedures. Improved purity of the harvested sample and heightened preadipocyte content are projected to provide for extended graft longevity.
\end{abstract}

\section{Keywords}

Laser Liposuction, Cell Viability, Fat Transfer, Preadipocyte

\footnotetext{
${ }^{*}$ Corresponding author.

How to cite this paper: Levenberg, A., Scheinowitz, M. and Sharabani-Yosef, O. (2015) Higher Cell Viability and Enhanced Sample Quality Following Laser-Assisted Liposuction versus Mechanical Liposuction. Journal of Cosmetics, Dermatological Sciences and Applications, 5, 238-245. http://dx.doi.org/10.4236/jcdsa.2015.53029
} 


\section{Introduction}

The dramatic evolution of contemporary plastic surgery has brought liposuction to become the fifth-most popular aesthetic procedure performed in Britain in 2014, with a 7\% rise in prevalence from the preceding year [1]. The procedure is performed to recontour defects of a spectrum of severities and, when harnessed toward autologous fat transfer applications, supports tissue reconstruction, radiation-induced necrosis of the chest wall, breast augmentation, volume enhancement in the facial area and wrinkle repair [2]. Autologous fat transfer circumvents complications associated with allogenic fillers and implants, is more readily available, more cost-effective, incurs minimal donor-site morbidity and provides a more durable outcome [3]. The constantly improving fat injection techniques have transformed autologous fat transfer into a minimally invasive, outpatient procedure.

However, highly variable resorption rates have been reported, averaging $45 \%$ graft weight retention within one year of transplantation [4] [5], where volume loss as high as 70\% has also been reported [6] [7], lending to overcorrection and reinjection sessions, and subsequent fat necrosis and calcification. Peer et al. established that the viable adipocyte content is the key determinant of fat transfer longevity [8]. Thus, minimization of the liquefied fat and serosanguinous fluid in the fat sample, increases the relative ratio of viable adipocytes, preventing early resorption as well as inflammatory reactions [9] [10]. Furthermore, while injection of fat specimens with high fibrous tissue content provides an immediate volumizing effect, postsurgical fibrosis positively correlates with adipocyte absorption and a short-lived clinical effect [11] [12]. Moreover, traces of blood, free oil fat and fibrotic tissue in transferred fat are said to accelerate adipocyte degradation [11], via increased inflammatory responses to the graft [13]. Thus, the ideal fat graft, containing a high adipocyte count and low contaminant content, has been the focus of harvesting optimization efforts for decades. Isolation solutions designed to maximize cell yield and viability, will inevitably ensure more durable clinical results and reduce the need for correction procedures.

As laser-assisted liposuction has often been charged with detrimental effects on cell viability, continuous efforts are being invested in design of a device that can maximize viable adipocyte yields. This study presents experience with a novel laser liposuction device featuring a $1470 \mathrm{~nm}$ diode laser and a radial emitting fiber. Specimen content and preadipocyte cell viability when harvested via laser-assisted lipolysis versus mechanical liposuctioning were compared. Laser-assisted liposuction proved more effective in preserving preadipocyte viability, while ensuring as fewer blood and connective tissue contaminations in the collected adipose tissue.

\section{Materials and Methods}

Donors: Human subcutaneous adipose tissue samples were obtained from the abdomen, thighs and inner thighs of 10 female subjects who had provided informed consent. All procedures were performed under general anesthesia and the average volume aspirated was 1.5 liters. Maximum aspired material was 3.5 liters. Minimum was 600 cc.

Surgical procedure: Patients were prepped with betadine. Saline, supplemented with lidocaine $20 \%$ (30 cc per liter saline) and adrenaline ( $0.5 \mathrm{ml}$ per liter saline) was introduced to the treated area via mechanical infusion (Byron Medical Inc.). Standard puncture holes were made at the treated areas with a \#11 surgical blade, to allow fat laser aspiration. The ratio of injected liquid (Tumescent) to aspirated material was 2:1. For fat aspiration, Mercedes $3 \mathrm{~mm}$ and $4 \mathrm{~mm}$ cannulas specially designed with a swivel handle (LipoLife, Alma Lasers) were used. The 1470 nm, 600 micron, radial emitting laser fiber (Alma Lasers, Ltd.) was advanced through the cannula and positioned in the center of the distal opening of the cannula.

Mechanical aspiration was then performed on the opposite side and by the same physician using $3 \mathrm{~mm}-4 \mathrm{~mm}$ Mercedes liposuction cannulas (Byron Medical Inc.). Temperature in the treated area was measured throughout the procedure and was maintained below $40^{\circ} \mathrm{C}$.

Adipose tissue harvesting: Fat samples were collected with a laser-assisted liposuction device (LipoLife, Al$\mathrm{ma}$ ) from one side of the patient and with a mechanical liposuction device (Byron) from the other side of the patient. Samples were not manipulated or washed in any way and were allowed to stand at room temperature to allow for phase separation. Samples were analyzed within 12 hours of collection.

Calculation of fat:blood phase ratios: The following formulation was applied to calculate the ratios between the phases into which specimens separated following liposuction: 


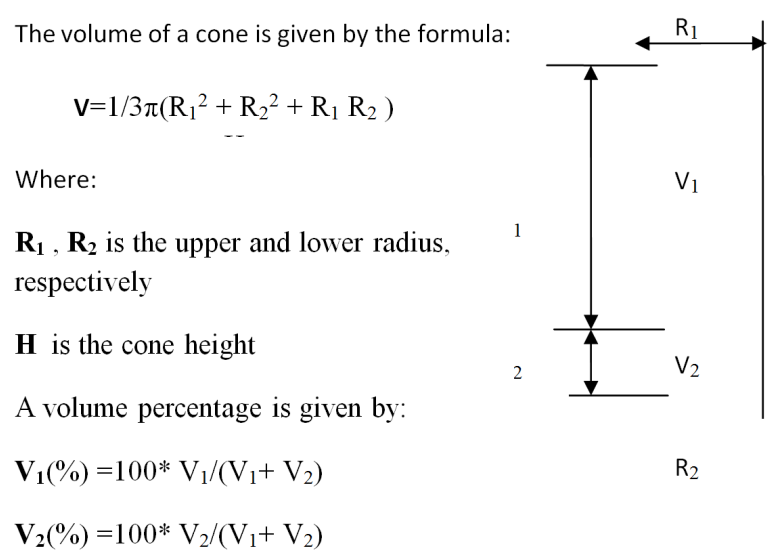

Cell yield, viability and morphology: Viable cell yield after isolation was determined using the trypan blue staining test. To assess the number of stem cells, the adherent cells were removed by proteolysis with trypsin $\mathrm{C}$ (Biological Industries, Israel). Cells were then stained with $0.4 \%$ trypan blue solution (10 $\mu \mathrm{l}$ cells: $10 \mu \mathrm{l}$ dye) and counted in a hemocytometer, viewed under a phase contrast microscope (Nikon). Duplicates samples from each specimen were evaluated. In addition, cell diameter, and connective tissue content were visually estimated.

Preadipocyte isolation: Preadipocytes were isolated after tissue harvesting. Fibrous structures and visible vessels were removed, and then washed up to seven times in phosphate-buffered saline (PBS) (Biological industries, Israel). After centrifugation (300 g, $10 \mathrm{~min}$ ) the tissue pellet was enzymatically digested with $2 \mathrm{mg} / \mathrm{mL}$ collagenase Type I (Sigma-Aldrich) dissolved in an equal volume of PBS solution $\left(37^{\circ} \mathrm{C}, 60 \mathrm{~min}\right)$. Collagenase was inactivated with 10\% fetal bovine serum (FBS) (Biological Industries, Israel), followed by redistribution of the mixture into $50 \mathrm{ml}$ conicals and centrifugation $(1000 \mathrm{~g}, 10 \mathrm{~min})$ to separate the oil and remaining fat lobules from the stromal vascular fraction (SVF). The red blood cells in the SVF pellet were then lysed in $160 \mathrm{mM}$ $\mathrm{NH} 4 \mathrm{Cl}$ (room temperature (RT), $10 \mathrm{~min}$ ). The sample was then washed twice in PBS and centrifuged (300 g, 5 min, RT). The adherent cell population was then isolated by culturing the cells overnight in flasks (DMEM F-12, $10 \%$ fetal calf serum, $2 \mathrm{mM}$ L-Glutamine, 0.1\% penicillin/streptomycin (Biological Industries, Israel)). The non-adherent cells and debris were washed away with PBS and the adipose stem cells were grown and expanded as monolayers. Cell viability was determined using trypan blue.

Statistical analysis: Comparative analyses between mechanical liposuction samples and laser liposuctioned samples were performed. Mean values and standard deviations are presented. Significance was determined using a one-sided Student's T-test.

\section{Results}

\subsection{Phase Separation of Collected Samples}

Samples harvested using laser lipolysis separated into two distinct phases, with a blood phase at the bottom of the canister, that contained only a small amount of blood, and a smooth, uniform, yellowish-pink adipose tissue phase at the top (Figure 1(a)). This latter phase consisted primarily of adipose cells, a minimal amount of connective tissue and very small lipid droplets (10 - $30 \mu \mathrm{m}$ diameter; Figure 2). Samples harvested by mechanical liposuction, separated into three distinct phases, which included, a blood phase at the bottom of the canister, adipose tissue phase in the middle and an oil phase at top (Figure 1(b)). The adipose tissue phase contained a significant amount of connective tissue, large adipose tissue fragments and large droplets (diameter $20 \mu \mathrm{m}$ - 100 $\mu \mathrm{m}$, Figure 2).

\subsection{Blood:Fat Ratios}

The blood, lipid droplets and adipose tissue phases of samples collected via mechanical liposuction comprised approximately $18 \%, 22 \%$ and $60 \%$ of the total sample, respectively. In contrast, laser liposuction samples separated into a $1.1 \%$ blood phase and $98.9 \%$ adipose tissue phase (Figure 1 ). These calculations were further supported by the significantly higher red blood cell content observed in samples collected by mechanical liposuc- 


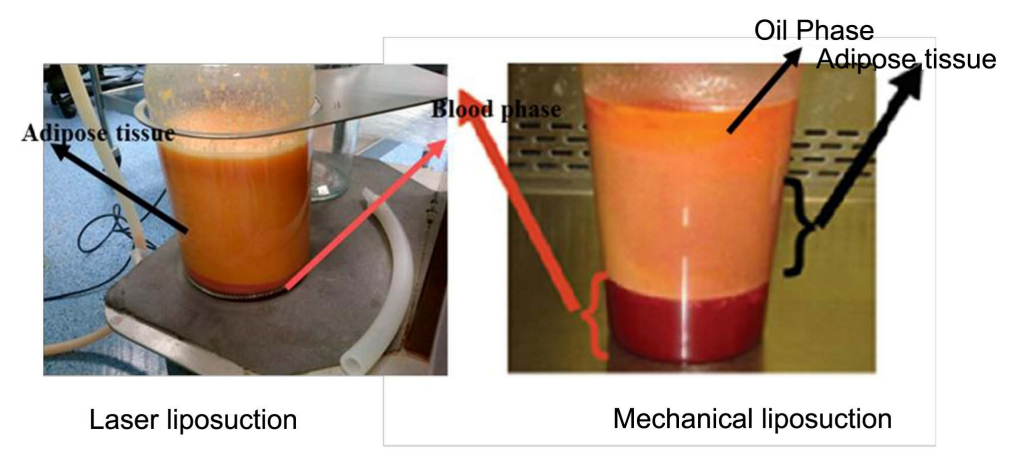

(a)

(b)

Figure 1. Human fat tissue aspirates Specimens were collected by (a) Laser lipolysis or (b) mechanical liposuction and separated into two and three phases, respectively.

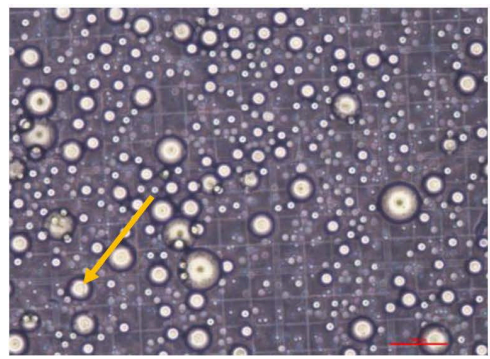

(a)

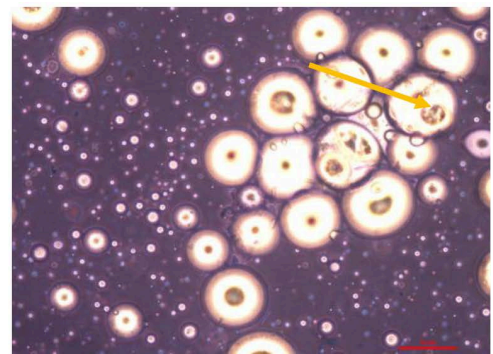

(b)

Figure 2. Microscopic analysis of the adipose phase of fat tissue aspirates Fat specimens were collected by (a) laser lipolysis or (b) mechanical liposuction and observed under a phase contrast microscope. The diameter of lipid droplets in the laser lipolysis-collected samples was much smaller than in mechanical liposuction samples (yellow arrows) (Magnification $\times 100$ ).

tion (Figure 3(b)).

\subsection{Cell Viability}

Cell viability in the tissue harvested by mechanical liposuction $(\mathrm{n}=9)$ was $79.7 \% \pm 18.3 \%$, while the laser liposuction samples $(\mathrm{n}=10$ ) consistently contained more viable cells, averaging $95.7 \% \pm 2.7 \%$ per sample (Figure 4, Table 1 , one-sided T-test $\mathrm{p}=0.005)$.

\subsection{Stem Cell Isolation from Adipose Tissue}

There were no significant differences in the number of viable stem cells isolated from adipose tissue harvested by way of laser liposuction vs. mechanical liposuction, although typical morphological features were better maintained among cells obtained by way of laser liposuction (Figure 5).

\subsection{Trauma Induced by Laser versus Mechanical Liposuction}

Laser liposuction decreased likelihood of burns and internal scarring, while the rounded cannula tip minimized tissue trauma (Figure 6) when compared to mechanical liposuction. Following mechanical liposuction, hemorrhages were still observed one week after treatment and more subcutaneous scarring was apparent.

\section{Discussion}

While no consensus has been reached regarding the best means of obtaining fat samples, it is largely agreed that long-term clinical effectiveness of adipose tissue grafts is heavily contingent upon the overall ratio of viable 


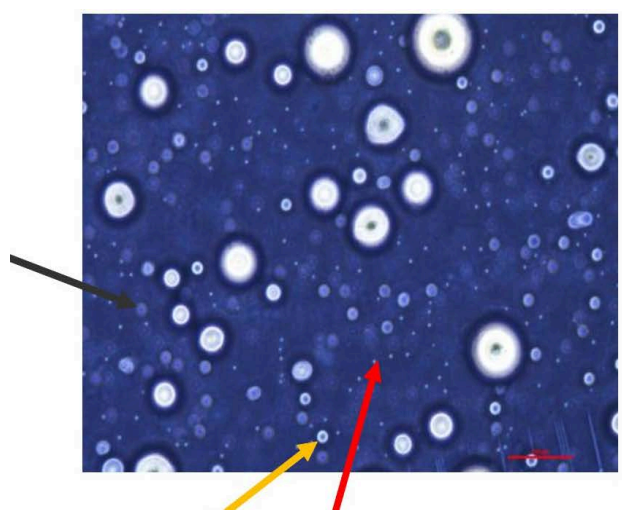

(a)

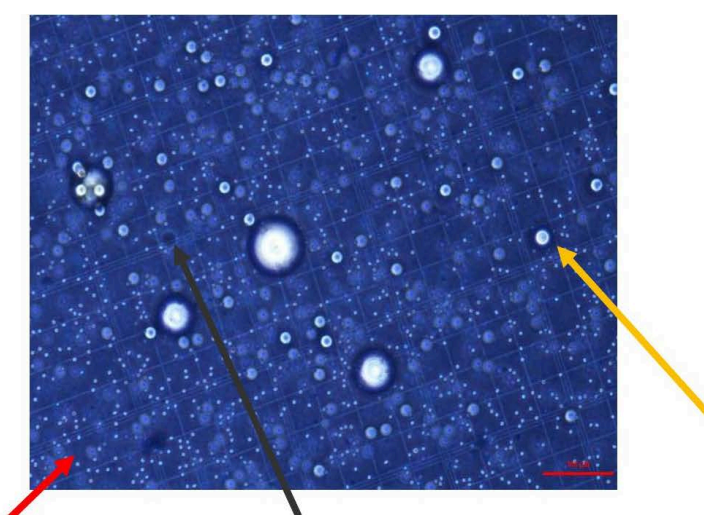

(b)

Figure 3. Adipose phase, trypan blue-stained specimens Fat specimens were collected by (a) laser lipolysis or (b) mechanical liposuction and stained with trypan blue before being observed and counted under a phase-contrast microscope. Viable cells (yellow arrows) have a clear cytoplasm whereas nonviable cells have a blue-stained cytoplasm (black arrows). Red blood cells are indicated by red arrows and were more prevalent in the mechanical liposuctioned specimens.

Table 1. Cell viability in the adipose phase of harvested adipose tissue samples.

\begin{tabular}{ccc}
\hline Sample \# & Laser & Mechanical \\
$\mathbf{1}$ & $98 \%$ & $96 \%$ \\
$\mathbf{2}$ & $96 \%$ & $92 \%$ \\
$\mathbf{3}$ & $96 \%$ & $91 \%$ \\
$\mathbf{4}$ & $95 \%$ & $50 \%$ \\
$\mathbf{5}$ & $96 \%$ & $66 \%$ \\
$\mathbf{6}$ & $99 \%$ & $88 \%$ \\
$\mathbf{7}$ & $95 \%$ & $55 \%$ \\
$\mathbf{8}$ & $96 \%$ & $90 \%$ \\
$\mathbf{9}$ & $96 \%$ & $89 \%$ \\
$\mathbf{1 0}$ & $90 \%$ & \\
Average & $95.7 \%$ & $79.7 \%$ \\
SD & 2.36 & 18.33 \\
\hline
\end{tabular}

adipocytes [8]. Over the years, the various liposuction techniques have been refined, in efforts to minimize mechanical and ischemic trauma, incidence of fat and pulmonary emboli, and reduced hemoglobin levels, and to enhance both short- and long-term specimen viability [14]. Laser-assisted liposuction induces both thermal and micromechanical lipolysis of the adipose tissue, improving skin contraction and reducing the need for traumatic aspiration forces and pressures and consequential patient pain [15], blood loss [16], hematomas and overall risk [17]. The added thermal component, overcomes the key limitation of mechanical liposuction, by melting connective tissue, coagulating vasculature, and stimulating neocollagen deposition, promoting skin contraction at the treatment site [17] [18].

The presented LipoLife device was designed with a $1470 \mathrm{~nm}$ diode laser, illuminating tissues at a wavelength effectively absorbed by cellular water, rendering it ideal for soft tissues by reducing the risk of tissue burn. In addition, its radial emitting fiber reduces the emission intensity in the surgical region, with decreased likelihood of burns and internal scarring, while the rounded cannula tip minimizes tissue trauma (Figure 5). In contrast, flat-tipped fibers deliver very high power densities, while the radial fiber delivers approximately 1/10 the power density.

This study demonstrated that laser liposuction of fat tissue with the LipoLife device, provides an ultimate and 


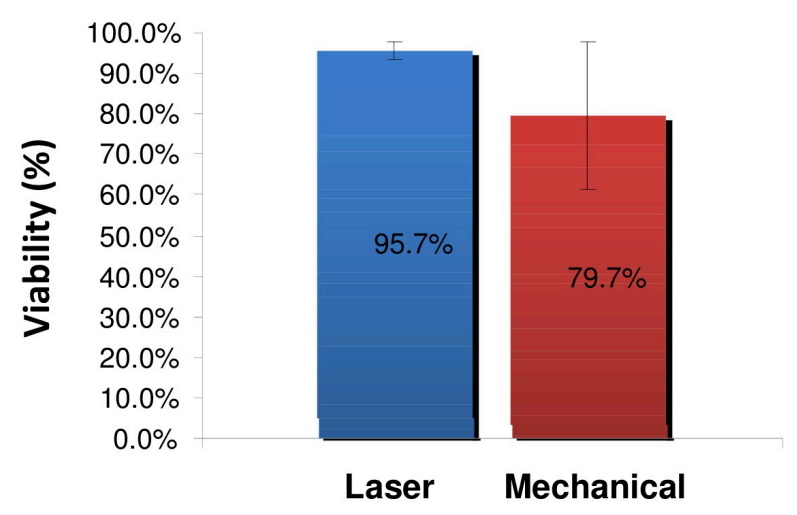

Figure 4. Cell viability in adipose phase samples Fat specimens were collected by laser lipolysis $(n=10)$ or mechanical liposuction $(n=9)$ and stained with trypan blue before being counted in a hemocytometer. The results represent the average of the samples \pm standard deviation.

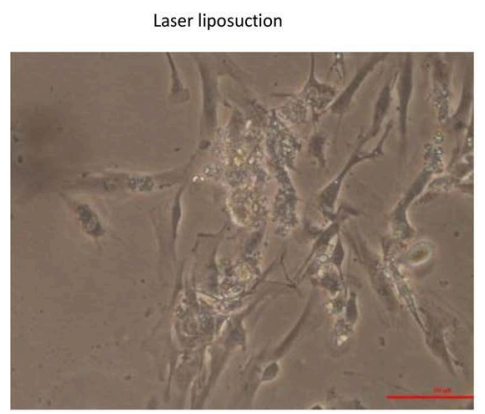

(a)

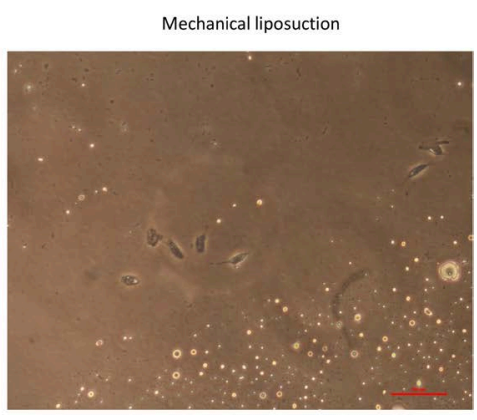

(b)

Figure 5. Morphology of stem cells isolated from adipose tissue collected by laser lipolysis versus mechanical liposuction Adipose tissue $(20 \mathrm{ml})$ was grown in a flask, as described in the methods section and photographed after three days in culture.

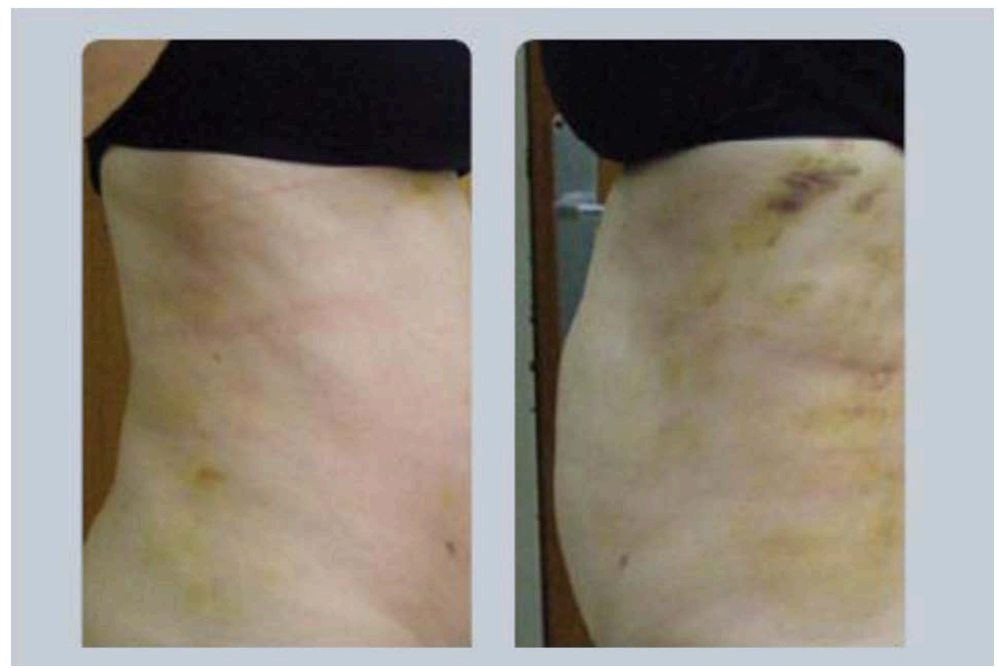

(a)

(b)

Figure 6. Trauma induced by laser versus mechanical liposuction Photographs taken one week after treatment with (a) laser liposuction and (b) mechanical liposuction. 
consistent source of preadipocytes, surpassing the quality and viability obtained by mechanical liposuction. The consistently higher cell yield, integrity and viability suggest that this extraction method avoids cell damage to the aspired adipose tissue. In addition, the low fibrous and blood, further promotes the clinical value of this harvesting method, by both minimizing patient risk and maximizing the potential of the preadipocyte specimen. Furthermore, the LipoLife laser liposuction harvesting yielded a more homogenous sample with less variable and more predictable results, as demonstrated by the uniform lipid droplet size and the lower standard deviations between samples, respectively. Overall, the improved purity of the harvested sample and heightened preadipocyte content, are projected to provide for extended graft longevity, reducing the incidence of overcorrection, and the need for repeat grafting and recontouring sessions. In addition, the high-quality samples bear significant potential in regenerative laboratories and in various clinical applications founded on the progenitor cell content in adipose tissue [19]. These early findings warrant further study of the functional advantage of the isolated specimens.

\section{References}

[1] http://www.plasticsurgerypractice.com/2015/01/baaps-data-plastic-surgery-falls-across-pond/

[2] Atiyeh, B., Costagliola, M., Illouz, Y.G., et al. (2015) Functional and Therapeutic Indications of Liposuction: Personal Experience and Review of the Literature. Annals of Plastic Surgery.

[3] Banyard, D.A., Salibian, A.A., Widgerow, A.D. and Evans, G.R. (2015) Implications for Human Adipose-Derived Stem Cells in Plastic Surgery. Journal of Cellular and Molecular Medicine, 19, 21-30. http://dx.doi.org/10.1111/jcmm.12425

[4] Peer, L.A. (1950) Loss of Weight and Volume in Human Fat Grafts. Plastic and Reconstructive Surgery, 5, 217. http://dx.doi.org/10.1097/00006534-195003000-00002

[5] Etzkorn, J.R., Divine, J.M., Lopez, J.J., et al. (2011) Autologous Fat Transfer: Techniques, Indications and Future Investigation. Cosmetic Dermatology, 24, 470-476.

[6] Kaufman, M.R., Miller, T.A., Huang, C., et al. (2007) Autologous Fat Transfer for Facial Recontouring: Is There Science behind the Art? Plastic and Reconstructive Surgery, 119, 2287-2296.

http://dx.doi.org/10.1097/01.prs.0000260712.44089.e7

[7] Fournier, P.F. (2000) Fat Grafting: My Technique. Dermatologic Surgery, 26, 1117-1128. http://dx.doi.org/10.1046/j.1524-4725.2000.00272.x

[8] Peer, L.A. (1956) The Neglected Free Fat Graft, Its Behavior and Clinical Use. American Journal of Surgery, 92, 40-44. http://dx.doi.org/10.1016/S0002-9610(56)80009-3

[9] Fagrell, D., Enestrom, S., Berggren, A., et al. (1996) Fat Cylinder Transplantation: An Experimental Comparative Study of Three Different Kinds of Fat Transplants. Plastic and Reconstructive Surgery, 98, 90-96, 97-98. http://dx.doi.org/10.1097/00006534-199607000-00014

[10] Coleman, S.R. (1995) Long-Term Survival of Fat Transplants: Controlled Demonstrations. Aesthetic Plastic Surgery, 19, 421-425. http://dx.doi.org/10.1007/BF00453875

[11] Sommer, B. and Sattler, G. (2000) Current Concepts of Fat Graft Survival: Histology of Aspirated Adipose Tissue and Review of the Literature. Dermatologic Surgery, 26, 1159-1166. http://dx.doi.org/10.1046/j.1524-4725.2000.00278.x

[12] Har-Shai, Y., Lindenbaum, E., Ben-Itzhak, O., et al. (1996) Large Liponecrotic Pseudocyst Formation Following Cheek Augmentation by Fat Injection. Aesthetic Plastic Surgery, 20, 417-419. http://dx.doi.org/10.1007/BF02390317

[13] Carpaneda, C.A. (1996) Study of Aspirated Adipose Tissue. Aesthetic Plastic Surgery, 20, 399-402. http://dx.doi.org/10.1007/BF02390314

[14] Heymans, O., Castus, P., Grandjean, F.X., et al. (2006) Liposuction: Review of the Techniques, Innovations and Applications. Acta Chirurgica Belgica, 106, 647-653.

[15] Prado, A., Andrades, P., Danilla, S., et al. (2006) A Prospective, Randomized, Double-Blind, Controlled Clinical Trial Comparing Laser-Assisted Lipoplasty with Suction-Assisted Lipoplasty. Plastic and Reconstructive Surgery, 118, 10321045. http://dx.doi.org/10.1097/01.prs.0000232428.37926.48

[16] Abdelaal, M.M. and Aboelatta, Y.A. (2014) Comparison of Blood Loss in Laser Lipolysis vs Traditional Liposuction. Aesthetic Surgery Journal, 34, 907-912. http://dx.doi.org/10.1177/1090820X14536904

[17] Badin, A.Z., Moraes, L.M., Gondek, L., et al. (2002) Laser Lipolysis: Flaccidity under Control. Aesthetic Plastic Surgery, 26, 335-339. http://dx.doi.org/10.1007/s00266-002-1510-3

[18] Goldman, A. (2006) Submental Nd:Yag Laser-Assisted Liposuction. Lasers in Surgery and Medicine, 38, 181-184. 
http://dx.doi.org/10.1002/lsm.20270

[19] Yoshimura, K., Shigeura, T., Matsumoto, D., et al. (2006) Characterization of Freshly Isolated and Cultured Cells Derived from the Fatty and Fluid Portions of Liposuction Aspirates. Journal of Cellular Physiology, 208, 64-76.

http://dx.doi.org/10.1002/jcp.20636 